\title{
Multiple ellipse fitting by center-based clustering
}

\author{
Tomislav Marošević ${ }^{1, *}$ and Rudolf Scitovski ${ }^{1}$ \\ ${ }^{1}$ Department of Mathematics, Josip Juraj Strossmayer University of Osijek \\ $\operatorname{Trg}$ Lj. Gaja 6, 31000 Osijek, Croatia \\ E-mail: 〈tmarosev, scitowsk@mathos.hr〉
}

\begin{abstract}
This paper deals with the multiple ellipse fitting problem based on a given set of data points in a plane. The presumption is that all data points are derived from $k$ ellipses that should be fitted. The problem is solved by means of center-based clustering, where cluster centers are ellipses. If the Mahalanobis distance-like function is introduced in each cluster, then the cluster center is represented by the corresponding Mahalanobis circle-center. The distance from a point $a \in \mathbb{R}^{2}$ to the Mahalanobis circle is based on the algebraic criterion. The well-known $k$-means algorithm has been adapted to search for a locally optimal partition of the Mahalanobis circle-centers. Several numerical examples are used to illustrate the proposed algorithm.
\end{abstract}

Key words: multiple ellipse fitting, center-based clustering, algebraic criterion, Mahalanobis distance

Received: October 1, 2014; accepted: December 13, 2014; available online: March 30, 2015

DOI:10.17535/crorr.2015.0004

\section{Introduction}

This paper considers multiple ellipses fitting problem based on a given set of data points. This problem appears in various areas, such as geology (strain estimation in rocks [25]), earthquake investigations [19], medicine [3, 6], robotics, object detection and other image processing industrial applications [12].

Suppose that the data set $\mathcal{A}=\left\{a_{i}=\left(x_{i}, y_{i}\right) \in \mathbb{R}^{2}: i=1, \ldots, m\right\} \subset \mathbb{R}^{2}$ is derived from $k$ ellipses that are to be fitted. Literature provides various approaches to solving this problem and is most often based on algebraic distance minimization $[9,12]$, least square distance minimization [1,21], arc-based approaches [15], or fuzzy clustering techniques $[2,6]$. On the other hand, methods based on the Hough transformation are used to solve the ellipse detection problem in digital images [12].

With the aim of solving this problem, a center-based clustering method [10, 11, 22 ] shall be applied in this paper. Set $\mathcal{A}$ will be partitioned into $k$ nonempty disjoint subsets $\pi_{1}, \ldots, \pi_{k}, 1 \leq k \leq m$, such that $\bigcup_{j=1}^{k} \pi_{j}=\mathcal{A}$. The partition will be denoted by $\Pi(\mathcal{A})=\left\{\pi_{1}, \ldots, \pi_{k}\right\}$. The elements of partition $\Pi$ are called clusters in $\mathbb{R}^{2}$, and the set of all such partitions are denoted by $\mathcal{P}(\mathcal{A} ; k)$. Data clustering is important

${ }^{*}$ Corresponding author. 
in a wide variety of applications such as medicine, biology, facility location problem, pattern recognition, information retrieval, understanding the Earth's climate, psychology, ranking of municipalities, etc. [3, 4, 8, 14, 17].

In order to apply the center-based clustering method, first it is necessary to define properly an ellipse-representative $E_{j}^{\star}$ corresponding to each cluster $\pi_{j} \in \Pi(\mathcal{A})$. The most natural way to do this is to solve the following global optimization problem (GOP)

$$
E_{j}^{\star} \in \underset{E \subset \mathbb{R}^{2}}{\operatorname{argmin}} \sum_{a \in \pi_{j}} D(a, E),
$$

where $D(a, E)$ is the distance from point $a$ to ellipse $E$ (an analogous definition for the circle-center is given in $[13,18])$.

If the Mahalanobis distance-like function is used (see, e.g., $[2,3,21]) d_{M}: \mathbb{R}^{2} \times$ $\mathbb{R}^{2} \rightarrow \mathbb{R}_{+}$,

$$
d_{M}(u, v ; S)=(u-v)^{T} S^{-1}(u-v),
$$

where $S \in \mathbb{R}^{2 \times 2}$ is a positive definite symmetric matrix, then an ellipse can be defined as a Mahalanobis circle (an M-circle)

$$
E(c, r ; S)=\left\{u \in \mathbb{R}^{2}: d_{M}(u, c ; S)=r^{2}\right\},
$$

where $c \in \mathbb{R}^{2}$ is a center, and $r>0$ the radius of an M-circle.

The M-circle (i.e., an ellipse) can be viewed as transformed circle, where matrix $S$ determining the major and the minor axes lengths, and the orientation of the ellipse [3].

Furthermore, in order to construct a modification of the $k$-means algorithm for searching for a locally optimal partition with M-circle-centers, the distance from a point $a \in \mathbb{R}^{2}$ to the ellipse $E(c, r ; S)$ should also be defined. For this purpose the following theorem from [2] is applied.

Theorem 1. Let $\|\cdot\|$ be a norm in $\mathbb{R}^{n}$ and let $B(c, r)=\left\{x \in \mathbb{R}^{n}:\|x-c\|=r, r>\right.$ $0\}$ be the closed ball of the radius $r$ centered at $c$. Then the shortest distance, as measured by $\|\cdot\|$, from a point $a \in \mathbb{R}^{n}$ to the ball $B(c, r)$ is given by $|\|a-c\|-r|$.

In applying Theorem 1 the LAD-distance from a point $a \in \mathbb{R}^{2}$ to the M-circle $E(c, r ; S)$ can be defined as

$$
D_{1}(a, E(c, r ; S))=\left|\sqrt{d_{M}(a, c ; S)}-r\right| .
$$

Specifically, the algebraic distance from a point $a \in \mathbb{R}^{2}$ to the M-circle $E(c, r ; S)$ can be defined as

$$
D(a, E(c, r ; S))=\left(d_{M}(a, c ; S)-r^{2}\right)^{2},
$$

which is often used in practical applications (see e.g. [9, 20]), and for this reason, the algebraic distance is used in this paper.

If each cluster $\pi_{j} \in \Pi$ is associated with its M-circle-center $E_{j}$ as defined by (1), then the problem of finding an optimal partition of the set $\mathcal{A}$ can be reduced to the following GOP (see, e.g., [2, 21, 23])

$$
\underset{\Pi \in \mathcal{P}(\mathcal{A} ; k)}{\operatorname{argmin}} \mathcal{F}(\Pi), \quad \mathcal{F}(\Pi)=\sum_{j=1}^{k} \sum_{a \in \pi_{j}} D\left(a, E_{j}\right) .
$$


Conversely, for a given set of different ellipses $E_{1}, \ldots, E_{k} \subset \mathbb{R}^{2}$, applying the minimum distance principle, the partition $\Pi=\left\{\pi_{1}, \ldots, \pi_{k}\right\}$ of the set $\mathcal{A}$ can be defined in the following way:

$$
\pi_{j}=\left\{a \in \mathcal{A}: D\left(a, E_{j}\right) \leq D\left(a, E_{s}\right), \forall s=1, \ldots, k, s \neq j\right\}, \quad j=1, \ldots, k .
$$

Therefore, the problem of finding an optimal partition of the set $\mathcal{A}$ can be reduced to the following optimization problem

$$
\underset{E_{1}, \ldots, E_{k} \subset \mathbb{R}^{2}}{\operatorname{argmin}} F\left(E_{1}, \ldots, E_{k}\right), \quad F\left(E_{1}, \ldots, E_{k}\right)=\sum_{i=1}^{m} \min _{j=1, \ldots, k} D\left(a_{i}, E_{j}\right) .
$$

The solutions from (5) and (7) coincide [21].

In general, function $F$ can have a large number of independent variables, need not be either convex or differentiable, but usually has several local and global minima. Hence, this becomes a complex GOP.

Section 2 describes a modification of the well-known $k$-means algorithm. The adaptive Mahalanobis distance-like function is introduced in Subsection 2.1 and adaptation of the $k$-means algorithm for searching a locally optimal partition is given in Subsection 2.2, where clusters are determined based on corresponding M-circlecenters. Subsection 2.2.1 considers the construction of a good initial approximation for the proposed algorithm. Finally, several illustrative examples are presented in Section 3.

\section{Modification of the $k$-means algorithm}

The well-known $k$-means algorithm deals with the search for a locally optimal $k$ partition of set $\mathcal{A}$. It is described in two steps that are repeated iteratively $[10,11]$.

Step A: For each set of mutually different centers $c_{1}, \ldots, c_{k} \in \mathbb{R}^{n}$, set $\mathcal{A}$ should be divided into $k$ disjoint clusters $\pi_{1}, \ldots, \pi_{k}$ using the minimum distance principle.

Step B: Given the partition $\Pi=\left\{\pi_{1}, \ldots, \pi_{k}\right\}$ of set $\mathcal{A}$, one can define the corresponding cluster centers.

The $k$-means algorithm depends strictly on the initial approximation of centers or initial partition. This algorithm can provide an acceptable solution, when a good initial approximation [11].

\subsection{Adaptive Mahalanobis distance-like function}

In modifying the $k$-means algorithm, the adaptive Mahalanobis distance-like function $d_{M}^{j}: \mathbb{R}^{2} \times \mathbb{R}^{2} \rightarrow \mathbb{R}_{+}$is defined for each cluster $\pi_{j}$ (see, e.g. $[2,3,14]$ )

$$
d_{M}^{j}\left(u, v ; S_{j}\right)=\sqrt{\operatorname{det} S_{j}}(u-v)^{T} S_{j}^{-1}(u-v),
$$

where

$$
S_{j}=\frac{1}{\left|\pi_{j}\right|} \sum_{a_{i} \in \pi_{j}}\left(a_{i}-c_{j}\right)\left(a_{i}-c_{j}\right)^{T}
$$


is a covariance matrix, $c_{j}$ is the centroid of the cluster $\pi_{j}$, and $\sqrt{\operatorname{det} S_{j}}$ is a normalized factor. The covariance matrix $S_{j}$ is usually a positive definite symmetric matrix. It is almost singular only in special cases, when the ellipse degenerates into a straight line or a point (i.e. one or two eigenvalues of $S_{j}$ are close to zero).

It can be shown that $[2,21]$

$$
c_{j}=\underset{x \in \mathbb{R}^{2}}{\operatorname{argmin}} \sum_{a \in \pi_{j}} d_{M}^{j}\left(a, x ; S_{j}\right)=\frac{1}{\left|\pi_{j}\right|} \sum_{a \in \pi_{j}} a .
$$

In particular, if $E_{j}\left(c_{j}, r_{j} ; S_{j}\right)$ is an M-circle in the cluster $\pi_{j}$, then the algebraic distance (4) from a point $a \in \mathcal{A}$ to the M-circle $E_{j}\left(c_{j}, r_{j} ; S_{j}\right)$ of the radius $r_{j}$ centered at $c_{j}$ is given by

$$
D\left(a, E_{j}\left(c_{j}, r_{j} ; S_{j}\right)\right)=\left(d_{M}^{j}\left(a, c_{j} ; S_{j}\right)-r_{j}^{2}\right)^{2}
$$

\subsection{Mahalanobis $k$-means}

To solve the multiple ellipses fitting problem, the previously described $k$-means algorithm is adapted to search for a locally optimal partition with M-circles as cluster centers.

In regard to Step $\mathrm{A}$, the distance from a data point $a \in \mathcal{A}$ to the M-circle-center is defined as the algebraic distance (11).

Subsequently, in Step B, the M-circle-center of the cluster $\pi_{j}$ is the solution to the following GOP

$$
\underset{\substack{c_{j} \in \mathbb{R}^{2}, r_{j} \in \mathbb{R}_{+} \\ S_{j} \in \mathbb{R}^{2 \times 2}}}{\operatorname{argmin}} \Phi_{j}\left(c_{j}, r_{j} ; S_{j}\right), \quad \Phi_{j}\left(c_{j}, r_{j} ; S_{j}\right)=\sum_{a \in \pi_{j}}\left(d_{M}^{j}\left(a, c_{j} ; S_{j}\right)-r_{j}^{2}\right)^{2},
$$

where $\Phi_{j}$ is the sum of algebraic distances from the points $a \in \pi_{j}$ to the M-circle $E_{j}\left(c_{j}, r_{j} ; S_{j}\right)$.

Now, the corresponding adapted $k$-means algorithm can be described in two steps, and are repeated iteratively.

Algorithm 1. (the $k$ closest M-circle-centers algorithm (KCMC))

Step A: For each set of mutually different $M$-circle-centers $E_{1}\left(c_{1}, r_{1} ; S_{1}\right), \ldots, E_{k}\left(c_{k}\right.$, $\left.r_{k} ; S_{k}\right)$ the set $\mathcal{A}$ should be divided into $k$ disjoint non-empty clusters $\pi_{1}, \ldots$, $\pi_{k}$ by using the minimum distance principle

$$
\begin{aligned}
& \pi_{j}=\left\{a \in \mathcal{A}: D\left(a, E_{j}\left(c_{j}, r_{j} ; S_{j}\right)\right) \leq D\left(a, E_{s}\left(c_{s}, r_{s} ; S_{s}\right)\right), \forall s=1, \ldots, k, s \neq j\right\}, \\
& j=1, \ldots, k .
\end{aligned}
$$

Step B: Given a partition $\Pi=\left\{\pi_{1}, \ldots, \pi_{k}\right\}$ of the set $\mathcal{A}$, the corresponding $M$-circlecenters $\hat{E}_{1}\left(\hat{c}_{1}, \hat{r}_{1} ; \hat{S}_{1}\right), \ldots, \hat{E}_{k}\left(\hat{c}_{k}, \hat{r}_{k} ; \hat{S}_{k}\right)$, can be defined, where

$$
\left(\hat{c}_{j}, \hat{r}_{j}, \hat{S}_{j}\right)=\underset{\substack{c_{j} \in \mathbb{R}^{2}, r_{j} \in \mathbb{R}_{+} \\ S_{j} \in \mathbb{R}^{2 \times 2}}}{\operatorname{argmin}} \sum_{a \in \pi_{j}} D\left(a, E\left(c_{j}, r_{j} ; S_{j}\right)\right) .
$$


According to [21, 23], the objective function (5), resp. (7), decreases monotonically. This ensures a normalized factor $\sqrt{\operatorname{det} S_{j}}$ incorporated into the definition of an adaptive Mahalanobis distance-like function (8).

As previously mentioned, the $k$-means algorithm is a method that generally provides a locally optimal partition. However, if a good initial approximation can be found, the $k$-means algorithm gives a satisfactory approximation of a globally optimal partition. Therefore, in Subsection 2.2.1 special attention is given to searching for an initial approximation of M-circle-centers which should be as best as possible.

To determine the M-circle centers in Step B of Algorithm 1, an appropriate initial approximation must first be defined for each cluster, i.e., the M-circle-center:

$$
\bar{E}_{j}=\left\{x \in \mathbb{R}^{2}: d_{M}^{j}\left(x, \bar{c}_{j} ; \bar{S}_{j}\right)=\bar{r}_{j}^{2}\right\} .
$$

The centroid of the set $\pi_{j}$ given by (10) is taken as an approximation of the center $\bar{c}_{j}$ of the M-circle-center and the matrix (9) is taken an approximation of covariance matrix $\bar{S}_{j}$. There still remains the requirement to construct a good approximation of the radius $\bar{r}_{j}$. For the given $\bar{c}_{j}, \bar{S}_{j}$, by definition

$$
r_{j}=\underset{r \in\left[0, R_{j}\right]}{\operatorname{argmin}} \sum_{a \in \pi_{j}} D\left(a, E\left(\bar{c}_{j}, r ; \bar{S}_{j}\right)\right)=\underset{r \in\left[0, R_{j}\right]}{\operatorname{argmin}} \sum_{a \in \pi_{j}}\left(d_{M}^{j}\left(a, \bar{c}_{j} ; \bar{S}_{j}\right)-r^{2}\right)^{2},
$$

where $R_{j}^{2}=\max _{a \in \pi_{j}} d_{M}\left(a, c_{j} ; S_{j}\right)$. Therefore, the initial approximation of the radius $\bar{r}_{j}$ is taken as the solution to the problem (14)

$$
\bar{r}_{j}^{2}=\frac{1}{\left|\pi_{j}\right|} \sum_{a \in \pi_{j}} d_{M}^{j}\left(a, \bar{c}_{j} ; \bar{S}_{j}\right) .
$$

After that, by knowing the initial approximation obtained in such way, for searching for the corresponding M-circle-centers in Step B we apply some locally optimization method (e.g., the Levenberg-Marquardt method or some other Newton-type method [7]).

Remark 1. It is well known (see, e.g., [2, 23, 24]) that this approach has certain drawbacks. When applying the $k$-means algorithm, the initial approximation should be defined, i.e., ellipses the set $\mathcal{A}$ belongs to should be ascertained and then these ellipses approximately determined. Furthermore, in the case of a small number of data points, the covariance matrix can be singular.

\subsubsection{Construction of a good initial approximation}

Let $\mathcal{A}$ be a set of data points that are, subject to some minor errors, likely to belong to $k$ ellipses. Indeed, the presumption is that the number and the position of the ellipses is unknown in advance.

When running Algorithm 1 (KCMC), the number and the position of possible ellipses should be estimated first. In order to do this, a visualization of the data set is necessary. For each ellipse, its five defining parameters are visually estimated: the 
center $c=(p, q)$, lengths of half-axes $a, b$, and the angle $\varphi$ between the half-axis $a$ and the positive direction of the $x$-axis. This ellipse is defined by the equation

$$
\frac{[(x-p) \cos \varphi+(y-q) \sin \varphi]^{2}}{a^{2}}+\frac{[-(x-p) \sin \varphi+(y-q) \cos \varphi]^{2}}{b^{2}}=1 .
$$

By means of the Mahalanobis distance-like function (2), ellipse (16) is expressed in the form of an M-circle

$$
E(c, 1 ; S)=\left\{u \in \mathbb{R}^{2}:(u-c)^{T} S^{-1}(u-c)=1\right\},
$$

with the radius equal to 1 , where $S$ is a symmetric positive definite matrix. It is relatively easy to show that the matrix $S$

$$
S=U\left[\begin{array}{cc}
a^{2} & 0 \\
0 & b^{2}
\end{array}\right] U^{T}, \quad \text { where } \quad U=\left[\begin{array}{cc}
\cos \varphi & -\sin \varphi \\
\sin \varphi & \cos \varphi
\end{array}\right] .
$$

Hence for $a, b>0$, the matrix $S$ is positive definite. It becomes easy to see that in the case of the normalized Mahalanobis distance-like function

$$
d_{M}(u, c ; S)=\sqrt{\operatorname{det} S}(u-c)^{T} S^{-1}(u-c),
$$

ellipse (16) is represented in the form of an M-circle with the radius $r^{2}=\sqrt{\operatorname{det} S}=$ $a b$, and the positive definiteness of the corresponding matrix is preserved. In this way, good initial approximations of M-circle-centers (i.e. approximations of their parameters $p, q, r, S)$ are obtained in the manner described above.

Remark 2. In a special case when an ellipse has half-axes a, $b$ parallel to the coordinate axes, the angle $\varphi=0$, and matrix (18) become diagonal, i.e., the matrix $S$ has the form $S=\operatorname{diag}\left(a^{2}, b^{2}\right)$.

Example 1. To illustrate a good initial approximation of an ellipse, the following example is given in Figure 1. Data points that come from ellipse (16) (the gray ellipse in Fig. 1a) with its respective parameters: $(p, q)=(4.8,4.3),(a, b)=(3.75,1.25)$, $\varphi=39^{\circ}$ are shown in Figure 1a. The initial approximation (the thin red ellipse in Fig. 1b) is obtained by visually estimating the ellipse parameters: center $c=(5,4)$,

\begin{tabular}{|c|c|c|c|c|c|c|c|c|}
\hline \multicolumn{3}{|c|}{ Original M-circle } & \multicolumn{3}{|c|}{ Initial approximation } & \multicolumn{3}{|c|}{ Solution } \\
\hline$\{p, q\}$ & $r$ & $S$ & $\{p, q\}$ & $r$ & $S$ & $\{p, q\}$ & $r$ & $S$ \\
\hline$(4.8,4.3)$ & 2.165 & {$\left[\begin{array}{ll}9.112 & 6.113 \\
6.113 & 6.513\end{array}\right]$} & $(5,4)$ & 2 & {$\left[\begin{array}{ll}8.5 & 7.5 \\
7.5 & 8.5\end{array}\right]$} & $(4.758,4.256)$ & 2.236 & {$\left[\begin{array}{ll}9.12 & 6.14 \\
6.14 & 6.63\end{array}\right]$} \\
\hline
\end{tabular}
half-axes $a=4, b=1$, and angle $\varphi=45^{\circ}$, and subsequently, the best-fit ellipse (12) (the thin red ellipse in Fig. 1c) is obtained. Table 1 gives parameter values of three $M$-circles, i.e., the original $M$-circle, the initial approximation and the solution.

Table 1: The original $M$-circle, the initial approximation and the best-fit $M$-circle 


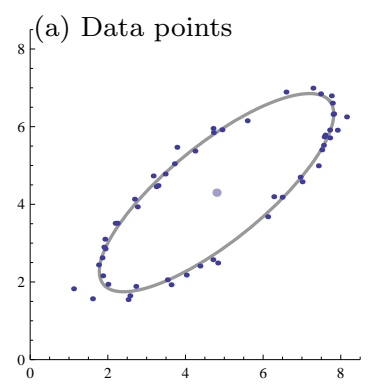

(b) Initial approximation

(c) Solution

Figure 1: Reconstruction of an ellipse

Remark 3. In accordance with Theorem 1, the multiple ellipse fitting problem based on the given set of data points in a plane could also be solved by using some other distance-like function. For example if the LAD-distance running from point $a \in \mathbb{R}^{2}$ to the $M$-circle $E(c, r ; S)$ is used (defined by $D_{1}(a, E(c, r ; S))=\mid \sqrt{d_{M}(a, c ; S)}-$ $r \mid)$, then in each cluster $\pi_{j}$, the $L A D$-distance from a point $a \in \mathcal{A}$ to the $M$-circle $E_{j}\left(c_{j}, r_{j} ; S_{j}\right)$ of the radius $r_{j}$ centered at $c_{j}$ is defined by

$$
D_{1}\left(a, E_{j}\left(c_{j}, r_{j} ; S_{j}\right)\right)=\left|\sqrt{d_{M}^{j}\left(a, c_{j} ; S_{j}\right)}-r_{j}\right| .
$$

Therefore, the construction of a good initial approximation of the radius $\bar{r}_{j}$, instead of (15) would require

$$
r_{j}=\underset{r \in\left[0, R_{j}\right]}{\operatorname{argmin}} \sum_{a \in \pi_{j}} D_{1}\left(a, E\left(c_{j}, r ; S_{j}\right)\right),
$$

and it follows that

$$
\bar{r}_{j}=\operatorname{med}_{a_{\in} \pi_{j}} \sqrt{d_{M}^{j}\left(a, c_{j} ; S_{j}\right)},
$$

where med is the median of $M$-distances from points $a \in \pi_{j}$ to the center $c_{j}$ of $M$-circle $E_{j}$.

In this way, it is also possible to modify the Mahalanobis $k$-means algorithm in the LAD sense. As expected, application of the LAD-distance function will show better behaviour when outliers are anticipated among the data (see [16]).

\section{Numerical examples}

This section provides a few numerical examples. Suppose that the number of clusters $k$ is given in advance in all the examples. Thereby, the set of data points are derived from $k$ ellipses that require reconstructing. In cases where number of clusters, i.e. ellipses, is not known in advance, various indexes could possibly be found that point to the most appropriate number of clusters in a partition (see e.g. [10, 18]).

Example 2. The set of four ellipses is given in parametric form

$$
E_{j}=c_{j}+\left(a_{x}^{(j)} \cos t, b_{y}^{(j)} \sin t\right), \quad t \in[0,2 \pi], \quad j=1,2,3,4,
$$


with the axes parallel to the coordinate axes (gray ellipses in Fig. 2). In the neighborhood of the $j$-th circle $m_{j} \sim \mathcal{U}(50,60)$ random points are generated by using binormal random additive errors with a mean vector $\mathbf{0} \in \mathbb{R}^{2}$ and the covariance matrix $0.1 \mathbf{I}$, where $\mathbf{I} \in \mathbb{R}^{2 \times 2}$ is the identity matrix. In this way, the data set $\mathcal{A}=\left\{a_{i} \in \mathbb{R}^{2}: i=1, \ldots, m\right\}$, with $m=58+50+55+59=222$ random points is obtained by using Gaussian distributions (see Fig. 2a). Ellipses should be reconstructed based on the data set $\mathcal{A}$.

Initial approximations of ellipses were obtained in the way described in Subsection 2.2.1 (thin red ellipses in Fig. 2b). Subsequently, by using Algorithm 1 and the Levenberg-Marquardt method for solving a nonlinear least squares problem, M-circlecenters (thin red ellipses in Fig. 2c that are almost covered with original gray ellipses) were obtained. The values $F$ of the corresponding sums of algebraic distances from the points to the M-circles are also shown in Fig. 2. On the basis of the values $F$, the visual view and the very small Hausdorff distance between the set of original ellipses and the set of obtained ellipses, the obtained $M$-circle-centers represent a good fit of the original ellipses.

(a) Data points $F=41.97$

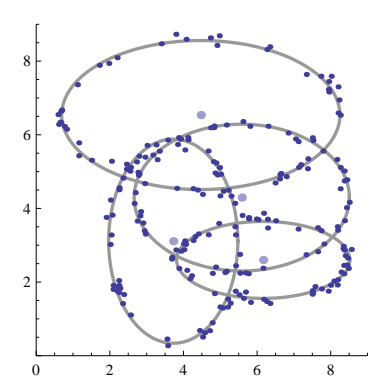

(b) Initial approximation $F=259.18$

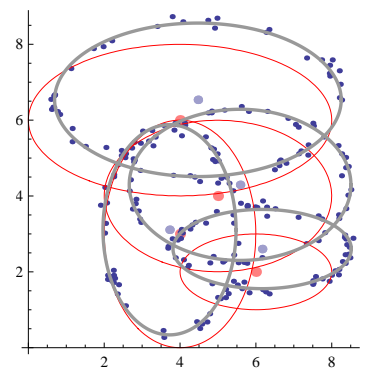

(c) Solution $F=34.52$

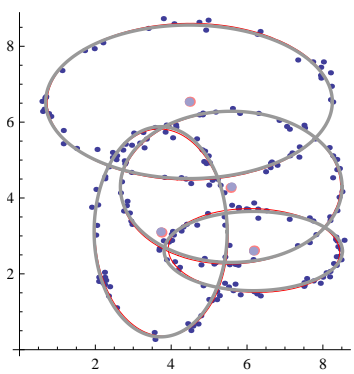

Figure 2: Mahalanobis k-means

Example 3. The set of four ellipses is given, two of which have axes that are not parallel to the coordinate axes (gray ellipses in Fig. 3). The corresponding data set $\mathcal{A}$ is constructed in a similar manner to Example 2 (see Fig. 3a). Ellipses are reconstructed based on the data set $\mathcal{A}$.

Initial approximations of ellipses were obtained in the manner described in Subsection 2.2.1 (see thin red ellipses in Fig. 3b). Thereafter, by using Algorithm 1 and the Levenberg-Marquardt method for solving a nonlinear least squares problem, $M$-circle-centers (thin red ellipses in Fig. 3c, that are almost covered by original gray ellipses) were obtained. Values $F$ of the corresponding sums of algebraic distances from the points to $M$-circles are also shown in Fig. 3. Based on the values $F$ and the visual inspection, the obtained $M$-circle-centers represent a good reconstruction of original ellipses. 
(a) Data points $F=108.85$

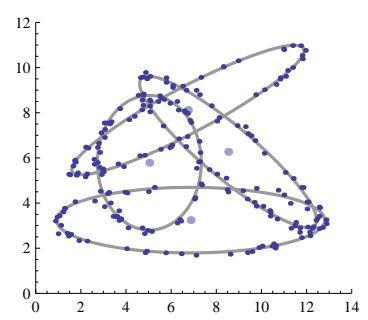

(b) Initial approximation $F=562.95$

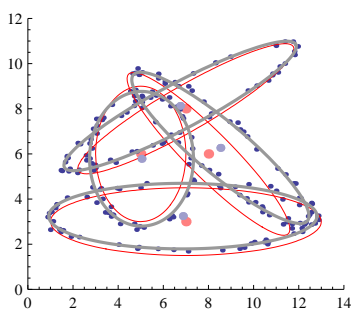

(c) Solution $F=87.22$



Figure 3: Mahalanobis $k$-means

Example 4. Consider the case shown in Fig. 4a. Note that the main axes of all ellipses are not parallel to the coordinate axes (see gray ellipses in Fig. 4).

Initial approximations of ellipses were obtained in the manner described in Subsection 2.2.1 (thin red ellipses in Fig. 4b). Hence, in based on Algorithm 1, using the Levenberg-Marquardt method for solving a nonlinear least squares problem, M-circlecenters (thin red ellipses in Fig. 4c, that are almost covered by gray original ellipses) were obtained. The values $F$ of the corresponding sums of algebraic distances from the points to the M-circles are also shown in Fig. 4. Based on the values $F$ and the visual view, these obtained $M$-circle-centers also represent a good fit of the original ellipses.

(a) Data points $F=67.12$

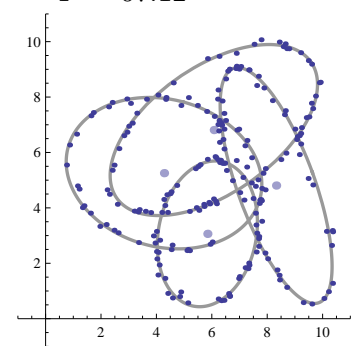

(b) Initial approximation $F=833.14$

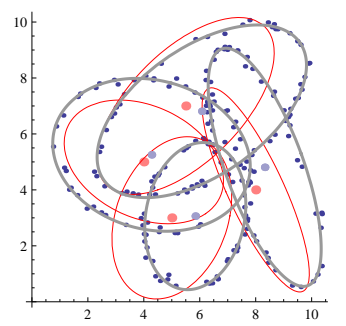

(c) Solution $F=56.75$

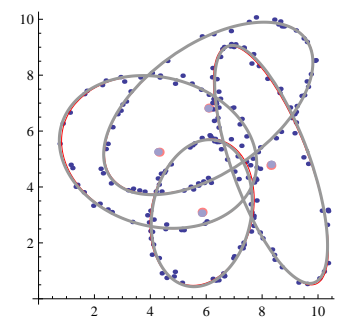

Figure 4: Mahalanobis k-means

Example 5. The case of three almost "concentric" ellipses with the same center and proportional axes is considered, as shown in Fig. 5 a.

Initial approximations of ellipses were obtained in the manner as described in Subsection 2.2.1 (thin red ellipses in Fig. 5b). Hence, in applying Algorithm 1, using the Levenberg-Marquardt method for solving nonlinear least squares problem, M-circle-centers (thin red ellipses in Fig. 5c that are almost covered by original gray ellipses) were obtained. The values $F$ of the corresponding sums of algebraic distances from the points to M-circles are also shown in Fig. 5. Based on the values $F$ and the visual view, these obtained $M$-circles also represent a good reconstruction of original ellipses. 



(c) Solution

$$
F=59.38
$$

Figure 5: Mahalanobis k-means

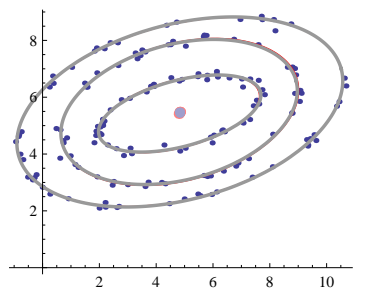

\section{Acknowledgements}

The authors would like to thank the anonymous referees and journal editors for their careful reading of the paper and their comments that helped in improving this paper. We are also thankful to Professor Kristian Sabo (Department of Mathematics, University of Osijek) for his useful comments and remarks.

\section{References}

[1] S.J.Ahn, W.Rauh, H.J.Warnecke, (2001). Least-squares orthogonal distances fitting of circle, sphere, ellipse, hyperbola, and parabola, Pattern Recognition, 34, 2283-2303. doi:10.1016/s0031-3203(00)00152-7.

[2] J.C. Bezdek, J.Keller, R.Krisnapuram, N.R.Pal, (2005). Fuzzy Models and Algorithms for Pattern Recognition and Image Processing. Springer, New York.

[3] B. Durak, (2011). A classification algorithm using mahalanobis distances of data with applications on biomedical data sets. Ph.D. thesis, The Graduate school of Natural and Applied Sciences of Middle East Technical University.

[4] G. Gan, C Ma, J. Wu, (2007). Data Clustering: Theory, Algorithms, and Applications. SIAM, Philadelphia.

[5] W. Gander, R. Strebel, G.H. Golub, (1995). Fitting of circles and ellipses least squares solution. SVD and Signal Processing III, 349-356. doi:10.1016/b978-0444821072/50036-4.

[6] I. Gath, D. Hoory, (1995). Fuzzy clustering of elliptic ring-shaped clusters. Pattern Recognition Letters, 16(7) 17, 727-741. doi:10.1016/0167-8655(95)00030-k.

[7] P.E. Gill, W. Murray, M.H Wright, (1981). Practical Optimization. Academic Press.

[8] Jain, J. (2010). Data clustering: 50 years beyond k-means. Pattern Recognition Letters, 31, 651-666. doi:10.1016/j.patrec.2009.09.011.

[9] K. Kanatani, P. Rangarajan, (2011). Hyper least squares fitting of circles and ellipses. Computational Statistics \& Data Analysis, 55(6), 2197-2208. doi:10.1016/j.csda.2010.12.012

[10] J. Kogan, (2007). Introduction to Clustering Large and High-Dimensional Data. Cambridge University Press.

[11] F. Leisch, (2006). A toolbox for k-centroids cluster analysis. Computational Statistics \& Data Analysis, 51, 526-544. doi:10.1016/j.csda.2005.10.006.

[12] Z. Liu, H. Qiao, (2009). Multiple ellipses detection in noisy environments: A hierarchical approach. Pattern Recognition, 42(11), 2421-2433. 
doi:10.1016/j.patcog.2009.01.028.

[13] T. Marošević, (2014). Data clustering for circle detection. Croatian Operational Research Review, 5(1), 15-24. doi:10.17535/crorr.2014.0025.

[14] A. Morales-Esteban, F. Martínez-Álvarez, S. Scitovski, R. Scitovski, (2014). A fast partitioning algorithm using adaptive Mahalanobis clustering with application to seismic zoning. Computers \& Geosciences, 73, 131-142. doi:10.1016/j.cageo.2014.09.003.

[15] Y. Qiao, S.H. Ong, (2007). Arc-based evaluation and detection of ellipses. Pattern Recognition, 40(7), 1990-2003. doi:10.1016/j.patcog.2006.10.009.

[16] K.Sabo, R. Scitovski, I. Vazler, (2013). One-dimensional center-based $l_{1}$-clustering method. Optimization Letters, 7(1), 5-22. doi:10.1007/s11590-011-0389-9.

[17] K. Sabo, R. Scitovski, I. Vazler, M.Zekić-Sušac, (2011). Mathematical models of natural gas consumption. Energy Conversion and Management, 52(3), 1721-1727. doi:10.1016/j.enconman.2010.10.037.

[18] R. Scitovski, T. Marošević, (2015). Multiple circle detection based on center-based clustering. Pattern Recognition Letters, 52, 9-16. doi:10.1016/j.patrec.2014.09.010.

[19] R. Scitovski, S. Scitovski, (2013). A fast partitioning algorithm and its application to earthquake investigation. Computers and Geosciences, 59, 124-131. doi:10.1016/j.cageo.2013.06.010.

[20] Q. Song et al., (2010). An information-theoretic fuzzy C-spherical shells clustering algorithm. Fuzzy Sets and Systems, 161(13), 1755-1733. doi:10.1016/j.fss.2009.11.004.

[21] H. Späth, (1983). Cluster-Formation und Analyse. R. Oldenburg Verlag, München.

[22] M. Teboulle, (2007). A unified continuous optimization framework for center-based clustering methods. Journal of Machine Learning Research, 8, 65-102.

[23] S. Theodoridis, K. Koutroumbas, (2009). Pattern Recognition. Acad. Press, Burlington.

[24] K.S. Younis, (1999). Weighted Mahalanobis distance for hyper-ellipsoidal clustering. Ph.D. thesis, Air Force Institute of Technology, Ohio.

[25] T.J. Wynn, S.A. Stewart, (2005). Comparative testing of ellipse-fitting algorithms: implications for analysis of strain and curvature. Journal of Structural Geology, 27(11), 1973-1985. doi:10.1016/j.jsg.2005.06.010. 